Internist 2021 · 62:121-122

https://doi.org/10.1007/s00108-020-00904-y

(c) Springer Medizin Verlag GmbH, ein Teil von Springer Nature 2021

Joachim Mössner

Medizinische Fakultät der Universität Leipzig, Leipzig, Deutschland

\title{
Polypen des Gastrointestinaltrakts
}

Ich freue mich, dass die Herausgeber der Zeitschrift und der wissenschaftliche Beirat einem Schwerpunkt zum Thema „Polypen des Gastrointestinaltrakts“ zugestimmt haben. Polypen sind häufige Befunde in der Gastroenterologie. Sie können zufällig bei einer Endoskopie entdeckt werden, ohne für die Symptomatik verantwortlich $\mathrm{zu}$ sein. In anderen Fällen wird gezielt nach ihnen gesucht, beispielsweise im Rahmen einer Vorsorgekoloskopie. Polypen können auch für die klinische Symptomatik verantwortlich sein und Blutungen oder einen Invaginationsileus bedingen. Im Internetlexikon Wikipedia steht zu lesen: „Das medizinische Wort Polyp ist rein deskriptiv (z. B. Nasenschleimhautpolyp - entzündlich; Dickdarmpolyp - neoplastisch) und sagt weder etwas über die Ätiologie noch etwas über die Dignität aus, d. h. ein neoplastischer Polyp kann sowohl gut- als auch bösartig sein. Die Differenzierung kann nur feingeweblich nach Abtragung erfolgen."

\section{\) Nichtneoplastische Polypen können auch malignes Potenzial haben}

I. Tischoff u. A. Tannapfel, Bochum, stellen die „Pathohistologie von Polypen des Gastrointestinaltrakts“ dar. Das Spektrum ist groß: entzündliche Polypen, Adenome, neuroendokrine Tumoren, gastrointestinale Stromatumoren, Fibrome, Leiomyome, Drüsenkörperzysten etc. Die neue Klassifikation der Weltgesundheitsorganisation von 2019 unterscheidet „Epitheliale und nichtepitheliale tumorartige Wandveränderungen von reaktiv entzündlich bis neoplastisch“. Nichtneoplastische Poly- pen können auch ein malignes Potenzial aufweisen, wie das Beispiel der hyperplastischen Polypen des Magens zeigt. Anhand der histologischen Eigenschaften lässt sich das maligne Potenzial bestimmen. Der Begriff der Dysplasie ist wieder gebräuchlich und ersetzt den Begriff der intraepithelialen Neoplasie.

I. Spier, R. Hüneburg u. S. Aretz, Bonn, geben in ihrem Beitrag "Gastrointestinale Polyposissyndrome "Einblick in die komplexe Molekulargenetik von Polypen. Unser Verständnis der unterschiedlichen molekularen Pathogenese von Malignomen basiert in vielen Aspekten auf der Erforschung der molekulargenetischen Veränderungen bei ererbten Polyposissyndromen. Ich möchte hier den Tumorsuppressor „adenomatosis polyposis coli“ (APC) nennen oder Mutationen einer Serin-Threonin-Kinase beim Peutz-Jeghers-Syndrom. Die Kanzerogenese bei der familiären Adenomatosis coli spiegelt in gewisser Weise im Zeitraffer die chronologisch auftretenden molekularen Veränderungen der Adenom-Karzinom-Sequenz wider. Nennen darf ich hier Onkogene wie KRAS, Tumorsuppressorgene wie APC oder TP53, Gene von Enzymen, die für die DNA-Reparatur verantwortlich sind, oder Gene, die die Apoptose regulieren.

B. Meier u. K. Caca, Ludwigsburg, besprechen die „Endoskopische Diagnostik, Therapie und Nachsorge von Polypen des oberen Gastrointestinaltrakts“. M. Hollenbach, J. Feisthammel u. A. Hoffmeister, Leipzig, befassen sich mit dem Thema „Endoskopische Diagnostik, Therapie und Nachsorge von Polypen des unteren Gastrointestinaltrakts“. Diese Beiträge haben in unterschiedlichen Aspekten klinische Relevanz. 
Muss jeder Polyp histologisch untersucht werden oder lässt sich makroskopisch Benignität mit Sicherheit feststellen? Im Rahmen der High-definitionEndoskopie stehen weitere Optionen zur Verfügung, unter anderem Vergrößerung, Chromoendoskopie, spezielle Darstellungen aufgrund von Einschränkungen der Lichtwellenbreite („narrow band imaging") und die Klassifikation des Polypen nach makroskopischen Aspekten („,pit pattern“).

Welches endoskopische Verfahren kommt bei welchen Polypen im oberen oder unteren Gastrointestinaltrakt zur Anwendung? Zu den möglichen Methoden zählen: Abtragung mit der elektrischen Schlinge mit oder ohne vorherige Adrenalinunterspritzung, Metallclip zur Blutungsprophylaxe, endoskopische Mukosaresektion, endoskopische Submukosadissektion, „kalte“ Schlingenabtragung, endoskopische Tunnelung und Vollwandresektion.

Eine ganz wesentliche Frage ist die Festlegung der endoskopischen Nachsorgeintervalle. Sie richtet sich nach dem histologischen Befund des primär abgetragenen Polypen. Ist das Übersehen von weiteren Polypen abhängig von der Zahl der primär endoskopisch gesehenen Polypen? Karzinome, die innerhalb von 10 Jahren nach einer Vorsorgekoloskopie auftreten, werden Intervallkarzinome genannt. Sie entstehen häufiger aus übersehenen Polypen, seltener handelt es sich um ein de novo entstandenes, schnell wachsendes Karzinom. Der Gastroenterologe kennt die Qualitätskriterien, die bei einer Vorsorgekoloskopie erfüllt sein müssen.

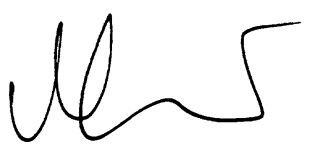

J. Mössner

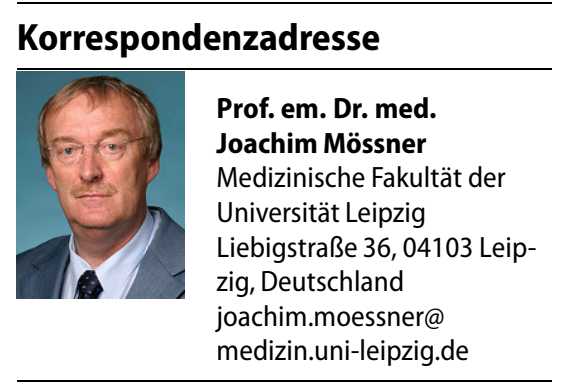

Prof. em. Dr. med. Joachim Mössner Ehemals Klinik und Poliklinik für Gastroenterologie und Rheumatologie, Department für Innere Medizin, Neurologie und Dermatologie, Universitätsklinikum Leipzig, AöR, Leipzig, Deutschland

Interessenkonflikt. J. Mössner gibt an, dass kein Interessenkonflikt besteht.

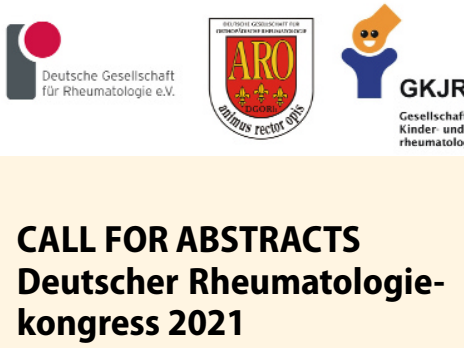

„Der besondere Fall"

Liebe Kolleginnen und Kollegen,

wir alle betreuen Patienten, deren Diagnose oder Verlauf durch ihre Seltenheit, die besondere Präsentation des Krankheitsfalles, ihren überraschenden Ausgang oder ihre innovative Therapieform besticht. Dieser Tatsache Rechnung tragend, will das Programmkomitee der DGRh für den kommenden Kongress in München das Forum „Der besondere Fall“ fortführen, das in den letzten Jahren großen Anklang gefunden hat.

Hiermit laden wir Sie ein, uns Ihre außergewöhnlichen Fälle in Abstract-Form zu beschreiben und einzureichen. Die Abstracts sollten gegliedert sein in:

1. Vorgeschichte

2. Leitsymptome bei

Krankheitsmanifestation

3. Diagnostik

4. Therapie

5. weiterer Verlauf

Im Titel soll die letztendliche Diagnose nicht genannt sein, um die Spannung zu erhalten.

Die Fälle können im Rahmen des Abstract-Verfahrens der DGRh bis 31.03.2021 online eingereicht werden.

Wir sind gespannt auf Ihre Beiträge und freuen uns auf eine neue und interessante Session in Nürnberg!

Prof. Dr. Georg Schett

DGRh-Kongresspräsident 2020

Prof. Dr. Hans-Dieter Carl

DGORh-Kongresspräsident 2020

Dr. Annette Holl-Wieden

Tagungsleiterin 2021 der GKJR 